\title{
Relação entre os parâmetros de rugosidade 3D e a molhabilidade do titânio com grãos micrométricos e sub-micrométricos
}

\author{
Relationship between 3D surface roughness \\ parameters and wettability in titanium with \\ micrometric and sub-micrometric grains \\ sizes
}

Gustavo Boehmer Leite ${ }^{1}$, Yuri Resende Fonseca ${ }^{2}$, Alaelson Vieira Gomes ${ }^{3}$, Carlos Nelson Elias ${ }^{4}$,

\author{
${ }^{1}$ Instituto Militar de Engenharia - Rio de Janeiro, RJ, Brasil. \\ ${ }^{2}$ Instituto Militar de Engenharia - Rio de Janeiro, RJ, Brasil. \\ ${ }^{3}$ Instituto Militar de Engenharia - Rio de Janeiro, RJ, Brasil. \\ ${ }^{4}$ Instituto Militar de Engenharia - Rio de Janeiro, RJ, Brasil. \\ e-mail: gustavoboehmer@gmail.com,yuriresende@yahoo.com.br, alaelson@ime.eb.br, elias@ime.eb.br
}

\section{RESUMO}

O titânio comercialmente puro (Ti cp) é o principal material usado na fabricação dos implantes dentários osseointegráveis. Para melhorar os índices de sucesso do tratamento odontológico e reduzir o tempo de osseointegração dos implantes, foram desenvolvidas várias metodologias de tratamento da superfície dos implantes. Para caracterizar as morfologias das superfícies dos implantes são usadas as análises no microscópio eletrônico de varredura (MEV), rugosidade, molhabilidade, identificação de possíveis contaminantes, ensaios in-vitro de culturas de células e in-vivo com animais. Entre as técnicas citadas, as mais usadas são as análises no MEV, as medidas dos parâmetros da rugosidade e da molhabilidade. Alguns trabalhos disponíveis na literatura sugerem a caracterização somente do valor de Ra sem apresentar uma explicação para a escolha deste parâmetro. No presente trabalho é feita a proposta de um modelo matemático para quantificar a relação entre os vários parâmetros da rugosidade 3D (Ra, Rq, Rz, Rms, Pico, Vale, PV, R3z e Smax) com a molhabilidade e a energia de superfície do Ti cp com dois tamanhos de grão (micrométricos e sub-micrométricos) e submetidos ao tratamento com ácido. Os resultados mostraram que para o nível de confiança de $95 \%$, as propriedades das superfícies do Ti cp tratados com ácido são mais influenciadas pelos parâmetros de rugosidade Ra e Rms.

Palavras-chave: titânio, rugosidade, molhabilidade, metodologia de LASSO

\begin{abstract}
Commercially pure titanium (cp Ti) is the main material used in the manufacture of dental implants. To improve the success rate of dental implant treatment some dental implant surface treatment methodologies have been developed. To characterize the surfaces of dental implants are used scanning electron microscope (SEM) analysis, roughness and wettability measurements, in-vitro cell culture and in-vivo animal experiments. Among the techniques cited, the simplest are SEM analyzes, of roughness and wettability measurements. Some papers available in the literature show values of surface roughness parameters and suggest the use of the Ra value without presenting any explanation for the choice of this parameter. In the present work, a mathematical model is proposed to quantify the relationship between some parameters of the 3D roughness (Ra, Rq, Rz, Rms, Peak, Vale, PV, R3z and Smax) with the wettability and surface energy of cp Ti with two grain sizes (micrometric and sub-micrometric). The results showed that the properties of the acid treated $\mathrm{cp}$ Ti surfaces are more influenced by the roughness parameters Ra and Rms.
\end{abstract}

Keywords: titanium, roughness, wettability, LASSO methodology

\section{INTRODUÇÃO}

Os implantes dentários osseointegráveis de titânio comercialmente puro (Ti cp) são utilizados como uma opção para restaurar as funções mastigatórias e melhorar a estética do paciente. O desempenho dos implantes depende de vários fatores, entre eles o material, a técnica cirúrgica, morfologia da superfície e local de inser- 
ção. Para aumentar a estabilidade mecânica inicial, melhorar as interações das células do organismo com a superfície dos implantes e reduzir o tempo de osseointegração foram desenvolvidos diversos tratamentos das superfícies dos implantes. A utilização de superfícies tratadas dos implantes dentários é uma forma de agilizar o tratamento odontológico. Com os tratamentos da superfície dos implantes dentários osseointegráveis é possível obter uma resposta mais favorável dos mecanismos que envolvem as interações das células e proteínas com a superfície dos implantes e com isto o processo de osseointegração torna-se mais eficiente [1]. Por meio do tratamento das superfícies com ácidos, jateamento, anodização ou combinações destes processos é possível obter boa interação das células do leito receptor com a superfície dos implantes. Dentre as propriedades que podem ser controladas com os tratamentos de superfície podemos citar a rugosidade, a energia da superfície e a molhabilidade. Esta última se refere à capacidade do líquido molhar o sólido, a qual é medida através do ângulo de contato do líquido com a superfície do material. As alterações advindas dos tratamentos das superfícies dos implantes modificam a adsorção de proteínas no biomaterial e, portanto a osseointegração $[2,3]$.

Para a caracterização as morfologias dos implantes após os tratamentos das superfícies são usadas análises no microscópio eletrônico de varredura (MEV). Na análise no MEV é possível verificar a qualidade do acabamento obtida na usinagem, a homogeneidade da rugosidade e a presença de impurezas. Os resultados do MEV são complementados pela análise da composição química, identificação do tipo de óxido por difração de raios-X, quantificação dos parâmetros da rugosidade, medido a molhabilidade e o potencial zeta. Quanto a medida dos parâmetros da rugosidade, a maioria dos trabalhos disponíveis na literatura caracteriza somente o parâmetro de rugosidade Ra. No entanto, além de Ra, outros parâmetros que caracterizam a rugosidade influenciam na interação das células com a superfície dos implantes [1,4].

A rugosidade da superfície pode ser quantificada por diversos parâmetros, como a média da rugosidade superficial (Ra), alturas dos picos, profundidades dos vales, áreas dos picos (Ap) e dos vales (Av), dentre outros parâmetros. A maioria dos trabalhos disponíveis na literatura que analisam a influência da rugosidade na osseointegração utiliza convencionalmente somente o valor de Ra. Outros trabalhos fazem a correlação do valor de Ra com outras propriedades das superfícies, entre elas, a molhabilidade, adesão das células in-vitro, adsorção de proteínas e índice de sucesso [3,4]. Estes trabalhos não justificam a escolha de Ra. Além disto, ainda permanece pouco esclarecida a forma como o parâmetro Ra influencia no sucesso e no tempo da osseointegração.

A avaliação isolada de um parâmetro da rugosidade de um implante não é a análise ideal para sua caracterização, pois superfícies com valores semelhantes de Ra podem apresentar morfologias diferenciadas [4]. Portanto, há necessidade de correlacionar entre si os diversos parâmetros que caracterizam a rugosidade, bem como analisar a interferência com outras propriedades da superfície dos implantes. As definições dos diversos parâmetros da rugosidade dos implantes podem ser encontradas na literatura [1].

No presente trabalho foi realizada a análise de diversos parâmetros usados para quantificar a rugosidade da superfície, e, através de um algoritmo obteve-se a correlação entre eles e com a molhabilidade. O objetivo foi identificar o parâmetro da rugosidade que exerce maior influência na molhabilidade e na energia da superfície. Com base nas análises realizadas é feita a proposta de um modelo matemático para quantificar a relação entre os parâmetros da rugosidade (Ra, Rq, Rz, Rms, Pico, Vale, PV, R3z e Smax) com a molhabilidade e a energia de superfície do Ti cp com dois tamanhos de grão (micrométricos e sub-micrométricos).

\section{MATERIAIS E MÉTODOS}

No presente trabalho foram utilizados discos de dois tipos Ti cp:

a) Ti ASTM Grau 4 (ASTM F67) no estado como recozido com tamanho de grãos micrométricos (Ti G4) e,

b) Ti ASTM Grau 4 (ASTM F67) submetido à deformação plástica severa ECAP para obter tamanho de grãos sub-micrométricos (Ti Hard) da ordem de $150 \mathrm{~nm}$ [5].

O Ti com tamanho de grãos sub-micrométricos recebe a denominação Ti Hard por ter sido endurecido pelo processo ECAP e apresentar resistência mecânica equivalente a do Ti ASTM grau 5. Os discos foram cortados das barras de 4,0 mm de diâmetro e submetidos aos tratamentos de superfície com duplo ataque

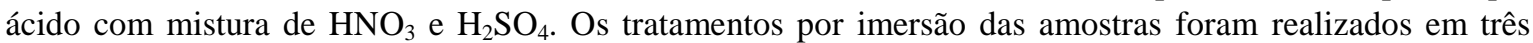
temperaturas $\left(\mathrm{T}_{1}=35^{\circ} \mathrm{C}, \mathrm{T}_{2}=40^{\circ} \mathrm{C}\right.$ e $\left.\mathrm{T}_{3}=45^{\circ} \mathrm{C}\right)$ e em três tempos $\left(\mathrm{t}_{1}=10 \mathrm{~min}, \mathrm{t}_{2}=15 \mathrm{~min}\right.$ e $\left.\mathrm{t}_{3}=20 \mathrm{~min}\right)$. As 
amostras foram divididas em 18 grupos (dois tamanhos de grãos, três temperaturas e três tempos de tratamento). superficial.

Após os tratamentos das superfícies dos discos mediu-se a rugosidade, molhabilidade e a tensão

Os parâmetros da rugosidade foram quantificados com o rugosímetro 3D Zygo modelo NewView 7100 profiler, o qual emprega a técnica de interferometria. Foram quantificados os parâmetros Ra, Rz, R3z, Rms, Pv, alturas dos picos, profundidades dos vales e Srmax em três áreas diferentes em cada amostra. Para cada condição de tratamento e tamanho de grão do Ti foram usados 3 discos.

A molhabilidade foi determinada com uso da técnica da gota d'água destilada. A tensão superficial e o ângulo de contato foram quantificados com a mesma metodologia com o emprego do goniômetro FTA100 FirstTenAngstron. Ao todo, oito parâmetros da rugosidade da superfície e duas medidas de molhabilidade foram quantificados.

Com os dados obtidos nos ensaios foi feita a proposta de um modelo matemático que relaciona as características da superfície da amostra com o tipo de Ti (Ti G4 e Ti Hard), com a molhabilidade e com a energia de superfície do material. O objetivo do modelo foi determinar a relação $\mathrm{f}: \mathrm{Rd} \times[0,1] \rightarrow \mathrm{R}$, onde $\mathrm{d}$ é o número de variáveis explicativas do modelo. No desenvolvimento do modelo matemático foi feita a hipótese que a relação entre as variáveis pode ser descrita pela equação:

$\mathrm{y}_{\mathrm{i}}=\delta_{\mathrm{i}}=(w)^{T} \mathrm{x}_{\mathrm{i}}+\mathrm{e}_{\mathrm{i}}+\mathrm{z}_{\mathrm{i}}$

onde:

$\mathrm{y}_{\mathrm{i}}$ é o ângulo de contato da amostra i

$(w)^{T} \mathrm{X}_{\mathrm{i}}$ são as observações das características da superfície (parâmetros de rugosidade)

$\mathrm{z}_{\mathrm{i}}$ é uma variável dummy que indica se a amostra é titânio Hard ou convencional (Ti G4), contida em $\mathrm{x}_{\mathrm{i}}$.

w é o vetor de coeficientes dos parâmetros de superfície.

$\delta$ é o possível efeito da molhabilidade do fato da amostra ser de Ti G4 ou Ti Hard.

$e_{\mathrm{i}}$ é uma expressão usada para minimizar o erro das variáveis.

Para otimizar a precisão da formulação proposta foi utilizada a técnica LASSO (Least Absolute Shrinkageand Selection Operator) [6]. A aplicação da técnica do LASSO pode ser formulada através do seguinte problema de otimização:

$w^{*}=\arg \min \frac{1}{N} \sum_{i=1}^{N}\left(y_{i}-(w)^{T} x_{i}\right)^{2}+\sum_{i=1}^{N} \lambda w_{i}$

onde $\mathrm{N}$ é o número de amostras, $|\mathrm{w}|_{1}$ é calculado pela soma dos valores absolutos dos elementos do vetor $w$ e $\lambda>0$, o qual é denominado constante de penalização do modelo. Normalmente, $|w|_{1}$ é escolhido através de um critério de informação como o BIC (Baysenian Information Criteria ou critério de informação bayseniano) ou AIC (Akaike Informatio Criteria ou critério de informação de Akaike).

A formulação da equação (2) se assemelha muito à formulação dos Mínimos Quadrados Ordinários (MQO), mas com a adição do termo $\lambda|w|{ }_{1}$. Como $\lambda>0$ e $|w|$ também é sempre maior do que zero, pois é a soma de valores absolutos, em um dado momento, adicionar mais variáveis explicativas na regressão para diminuir o erro de previsão (termo do somatório) não compensa devido ao aumento do número $|w|_{1}$. Desta forma, utilizando a técnica acima, o algoritmo do LASSO será capaz de identificar os parâmetros da rugosidade da superfície que são mais importantes para estimar a molhabilidade, dado o pequeno número de amostras. Com esta metodologia, as variáveis menos relevantes serão forçadas a serem iguais a zero para evitar o aumento do termo $\lambda|w|_{1}$. No entanto, sabe-se que quando aplicada qualquer técnica de seleção de modelos, seja ela stepwise regression, LASSO ou qualquer outra metodologia, pode-se chegar a resultados errôneos pelo chamado viés de seleção de modelo. Para realizar inferência estatística sobre os coeficientes do modelo (1), foi utilizada a técnica de Double Selection apresentada por BELLONI et al. [7].

O método Double Selection é aplicado da seguinte forma:

a) Escolhe-se a variável em que se deseja realizar a inferência (Ra por exemplo);

b) Resolve-se o problema (2) utilizando como variável y como sendo a molhabilidade e as demais variáveis como explicativas, exceto a selecionada na etapa anterior (exclui-se Ra);

c) Resolve-se o problema (2) utilizando agora como y a variável selecionada na primeira etapa (Ra) e as demais como explicativas; 
d) Ajusta-se o modelo final por regressão linear utilizando como y a variável molhabilidade e como variáveis explicativas somente as selecionadas por LASSO nas etapas 1, 2 e 3.

A sequência apresentada deve ser repetida para todas as variáveis que se deseja realizar a inferência. Após a aplicação do método de Double Selection, a inferência final pode ser feita de forma convencional, com o teste t-student por exemplo.

\section{RESULTADOS}

Mostra-se na Figura 1 exemplos das morfologias das superfícies das amostras obtidas com o rugosímetro Zygo. A escala de cores indica a presença das irregularidades na superfície na forma de reentrâncias ou saliências. A cor vermelha indica a presença de picos e cor verde indica a presença de cavidades na superfície.

Mostra-se nas Tabelas 1 e 2 os parâmetros da rugosidade, a molhabilidade medida pelo ângulo de contato de uma gota d'água e a energia da superfície determinada pela tensão superficial das amostras de Ti G4 e Ti Hard.

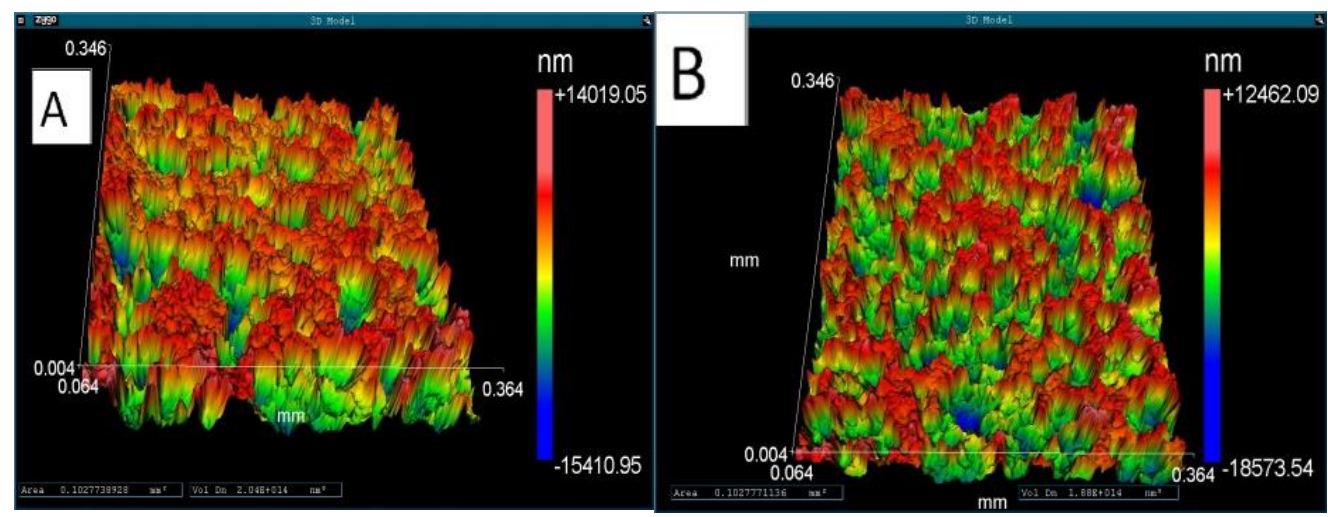

Figura 1: Morfologias típicas das superfícies obtidas com o rugosímetro 3D Zygo. (A) Ti G4. (B) Ti Hard.

Tabela 1: Parâmetros da rugosidade, molhabilidade e tensão superficial das amostras de Ti G4.

\begin{tabular}{c|c|c|c|c|c|c|c|c|c|c}
\hline Tratamento & $\begin{array}{c}\text { Ra } \\
(\boldsymbol{\mu m})\end{array}$ & $\begin{array}{c}\text { PV } \\
(\boldsymbol{\mu m})\end{array}$ & $\begin{array}{c}\text { Rms } \\
(\boldsymbol{\mu m})\end{array}$ & $\begin{array}{c}\text { Pico } \\
(\boldsymbol{\mu m})\end{array}$ & $\begin{array}{c}\text { Rz } \\
(\boldsymbol{\mu m})\end{array}$ & $\begin{array}{c}\text { Vale } \\
(\boldsymbol{\mu m})\end{array}$ & $\begin{array}{c}\text { R3z } \\
(\boldsymbol{\mu m})\end{array}$ & $\begin{array}{c}\text { Srmax } \\
(\boldsymbol{\mu m})\end{array}$ & $\begin{array}{c}\hat{A} g u l o \\
\text { contato } \\
(\mathbf{g r a u})\end{array}$ & $\begin{array}{c}\text { Tensão su- } \\
\mathbf{p e r f i c i a l} \\
(\mathbf{n N} / \mathbf{m})\end{array}$ \\
\hline T1t1 & 0,46 & 45,95 & 0,65 & 22,69 & 24,07 & $-23,26$ & 19,51 & 32,00 & 118,12 & $-34,29$ \\
\hline T1t2 & 0,75 & 36,95 & 1,07 & 19,37 & 25,81 & $-17,58$ & 24,37 & 27,31 & 104,27 & $-17,98$ \\
\hline T1t3 & 0,75 & 27,87 & 0,99 & 10,07 & 18,67 & $-17,81$ & 17,61 & 27,87 & 116,45 & $-31,95$ \\
\hline T2t1 & 1,10 & 54,59 & 1,62 & 32,22 & 48,56 & $-22,37$ & 49,70 & 51,03 & 126,83 & $-43,75$ \\
\hline T2t2 & 1,05 & 55,74 & 1,36 & 38,45 & 45,54 & $-17,29$ & 48,45 & 45,99 & 114,20 & $-29,90$ \\
\hline T2t3 & 1,41 & 54,59 & 1,95 & 36,00 & 51,93 & $-18,59$ & 52,00 & 50,95 & 120,40 & $-36,78$ \\
\hline T3t1 & 2,94 & 62,91 & 3,97 & 32,16 & 52,15 & $-30,75$ & 54,63 & 51,13 & 129,27 & $-46,19$ \\
\hline T3t2 & 2,92 & 42,16 & 4,08 & 11,94 & 38,89 & $-30,22$ & 38,40 & 40,59 & 132,51 & $-49,32$ \\
\hline T3t3 & 4,44 & 89,59 & 6,17 & 42,61 & 79,02 & $-46,98$ & 78,06 & 82,64 & 134,84 & $-51,34$ \\
\hline
\end{tabular}

Tabela 2: Parâmetros da rugosidade, molhabilidade e tensão superficial das amostras de Ti Hard.

\begin{tabular}{c|c|c|c|c|c|c|c|c|c|c|}
\hline Tratamento & $\begin{array}{c}\text { Ra } \\
(\boldsymbol{\mu m})\end{array}$ & $\begin{array}{c}\text { PV } \\
(\boldsymbol{\mu m})\end{array}$ & $\begin{array}{c}\text { Rms } \\
(\boldsymbol{\mu m})\end{array}$ & $\begin{array}{c}\text { Pico } \\
(\boldsymbol{\mu m})\end{array}$ & $\begin{array}{c}\text { Rz } \\
(\boldsymbol{\mu m})\end{array}$ & $\begin{array}{c}\text { Vale } \\
(\boldsymbol{\mu m})\end{array}$ & $\begin{array}{c}\text { R3z } \\
(\boldsymbol{\mu m})\end{array}$ & $\begin{array}{c}\text { SRmax } \\
(\boldsymbol{\mu m})\end{array}$ & $\begin{array}{c}\text { Angulo } \\
\text { contato }\end{array}$ & $\begin{array}{c}\text { Tensão su- } \\
\mathbf{p e r f i c i a l} \\
(\mathbf{n N} / \mathbf{m})\end{array}$ \\
\hline T1t1 & 0,57 & 62,59 & 0,74 & 40,31 & 32,44 & $-22,27$ & 23,35 & 45,37 & 111,32 & $-26,32$ \\
\hline T1t2 & 0,45 & 51,18 & 0,73 & 14,41 & 33,94 & $-36,77$ & 28,06 & 48,18 & 120,35 & $-36,88$ \\
\hline T1t3 & 0,94 & 28,08 & 1,17 & 14,13 & 23,37 & $-13,94$ & 23,48 & 24,62 & 125,29 & $-42,14$ \\
\hline T2t1 & 0,94 & 28,08 & 1,17 & 14,13 & 23,37 & $-13,94$ & 23,48 & 24,62 & 120,32 & $-36,85$ \\
\hline T2t2 & 1,00 & 60,83 & 1,49 & 31,70 & 54,94 & $-29,13$ & 56,56 & 59,69 & 116,74 & $-32,84$ \\
\hline T2t3 & 0,99 & 56,84 & 1,35 & 30,82 & 48,73 & $-26,02$ & 47,95 & 43,28 & 111,66 & $-26,93$ \\
\hline
\end{tabular}




\begin{tabular}{l|l|l|l|l|l|l|l|l|l|l|}
\hline T3t1 & 3,10 & 50,71 & 4,30 & 18,47 & 43,26 & $-32,24$ & 41,72 & 40,38 & 109,21 & $-11,68$ \\
\hline T3t2 & 5,70 & 50,91 & 7,60 & 18,59 & 43,14 & $-32,32$ & 41,85 & 46,92 & 127,37 & $-44,29$ \\
\hline T3t3 & 7,09 & 94,61 & 9,87 & 44,84 & 93,03 & $-49,77$ & 93,17 & 92,52 & 128,74 & $-45,60$ \\
\hline
\end{tabular}

Na figura 2 é mostrada a relação entre a tensão superficial e o ângulo de contato da gota d'água com a superfície de titânio. Pode-se observar que existe uma relação inversamente proporcional entre as duas propriedades, independendo se as amostras possuem tamanho de grão micrométrico (Ti G4) ou submicrométrico (Ti Hard).

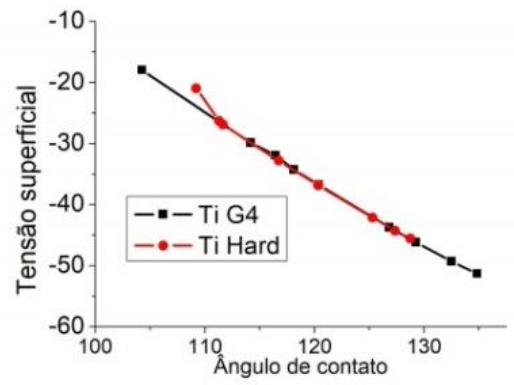

Figura 2: Variação da tensão superficial com o ângulo de contato (molhabilidade).

Mostra-se na figura 3 a influência dos parâmetros da rugosidade Ra e Rms no ângulo de contato. Os resultados da Figura 3 mostram que a relação entre a rugosidade e a molhabilidade não é linear. Este resultado justifica a proposta do emprego de nova metodologia de análise da influência dos parâmetros da rugosidade na molhabilidade.

Na figura 4 é mostrada a variação do ângulo de contato com a altura do pico, valores de Rz, R3z e Srmax. Pode-se observar nestas figuras que o ângulo de contato apresenta forte dependência com os parâmetros da rugosidade. No entanto, a relação entre os parâmetros da rugosidade e a molhabilidade não é linear. A influência dos parâmetros da rugosidade na molhabilidade é confirmada pelos dados da análise estatística que são apresentados na Tabela 3.

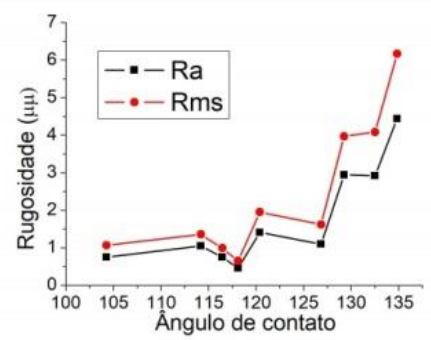

Figura 3: Variação da tensão superficial com os parâmetros de rugosidade Ra, Rms, pico, Rz, R3z e Srmax.

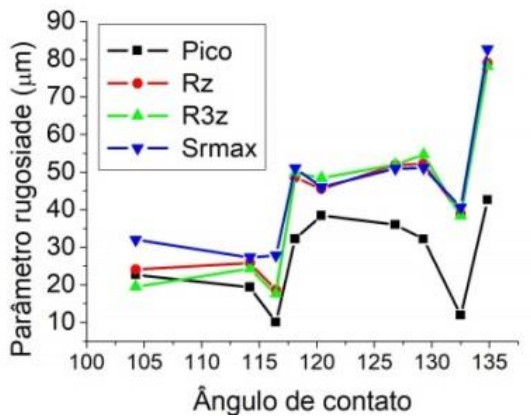

Figura 4: Variação do ângulo de contato com a altura do pico, valores de Rz, R3z e Srmax.

Os dados da Tabela 3 mostram que para o nível de significância de 95\%, quanto menor o valor de pvalue, maior a influência da variável.

Nas análises estatísticas convencionais ou mesmo empregando a metodologia de Análise de experimentos, Análise fatorial ou Análise de Yates, a hipótese é que a influência dos parâmetros é linear, bem como as interações entre as variáveis. Nestas análises, para visualizar a comparação dos efeitos das variáveis 
devemos eleger dois parâmetros e representá-los em gráficos tri-dimensionais. Na metodologia usada no presente trabalho, as influências dos diversos parâmetros e as interações entre eles são analisados simultaneamente, maximizando ou minizando os efeitos.

Na Tabela 3, os valores estimados e que foram obtidos nas simulações são baixos e foram arredondados para zero. Igualmente, os desvios padrões por serem baixos foram arredondados. A grande diferença nos resultados estimados dos diversos parâmetros deve-se ao fato que a escala das variáveis não está normalizada. Em consequência, alguns parâmetros possuem coeficientes e desvios muito grandes e outros são pequenos.

Tabela 3: Parâmetros obtidos na análise estatística. Analisando o $p$-value da coluna Pr, todos os valores menores que 0,01 indicam que as variáveis são importantes. Quanto menor o $p$-value mais importante é a variável.

\begin{tabular}{l|l|l|l|l}
\hline & Estimativa & Erro padrão & Valor $\mathbf{t}$ & $\operatorname{Pr}(>|\mathbf{t}|)$ \\
\hline $\mathrm{Ra}$ & 115,91 & 49,98 & 2,32 & 0,02 \\
\hline $\mathrm{Pv}$ & $-6809,93$ & 16511,40 & $-0,41$ & 0,68 \\
\hline $\mathrm{Rms}$ & $-86,19$ & 35,64 & $-2,42$ & 0,02 \\
\hline Pico & 0,0 & 0,0 & 0,25 & 0,81 \\
\hline $\mathrm{Rz}$ & 0,0 & 0,0 & $-1,49$ & 0,14 \\
\hline Vale & 0,0 & 0,0 & $-1,71$ & 0,09 \\
\hline R3z & 0,0 & 0,0 & $-1,91$ & 0,06 \\
\hline Srmax & 0,0 & 0,0 & 0,31 & 0,76 \\
\hline Tamanho grão & 5,62 & 9,13 & 0,62 & 0,54 \\
\hline
\end{tabular}

Considerando o nível de significância de 95\%, os resultados obtidos pelo modelo proposto no presente trabalho indicam que somente os parâmetros da rugosidade Ra e o Rms são suficientes para prever a molhabilidade. Considerando o nível de significância mais flexível de $90 \%$, é possível predizer a molhabilidade do titânio medido os valores de Ra, Rms, Vale e R3z. Os resultados obtidos mostram que não é necessário medir todos os parâmetros da rugosidade para prever a molhabilidade.

Mostra-se na figura 5 o resultado da análise da interdependência entre os vários parâmetros da rugosidade usando o algoritmo proposto no presente trabalho. Para interpretar a figura deve-se considerar:

a) As dimensões dos círculos: quanto maior o diâmetro do círculo na interseção da linha com a coluna, maior a dependência entre os dois parâmetros da rugosidade.

b) A cor: círculos com diâmetros iguais indicam que as influencias dos parâmetros são iguais mas, pode ter influência positiva quanto mais próxima da cor azul escura ou negativa quanto mais próxima da cor vermelha.

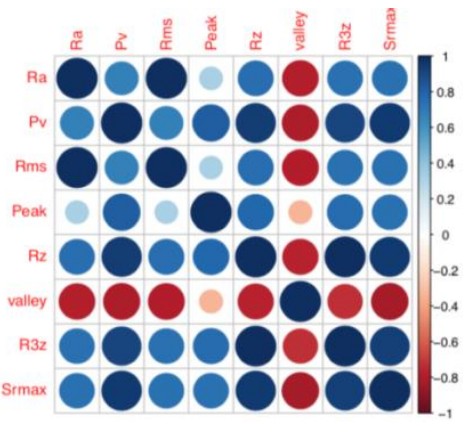

Figura 5: Resultado da análise da interdependência entre os parâmetros da rugosidade. A dimensão dos círculos esta diretamente proporcional à interdependência entre os parâmetros da rugosidade.

\section{DISCUSSÃO}

A metodologia da análise de dados e o uso do algoritmo proposto indicaram que existe uma forte interdependência entre os valores quantificados da rugosidade com a molhabilidade (Fig 1 e 2). Esta interdependência 
linear também é citada na literatura [4]. YAN et al. [8] compararam as influencias da rugosidade de amostras de Ti-6Al-4V polidas e com ataque ácido. Os autores empregaram várias equações para calcular a energia da superfície e analisaram a influencia da rugosidade. Os autores concluíram que não existe uma relação entre os parâmetros de rugosidade $\mathrm{Ra}, \mathrm{Rq}$ e Rt com a energia de superfície aparente. Resultado oposto ao do presente trabalho.

No presente trabalho os ângulos de contato de todas as amostras foram maiores que 90 graus. Isto permite classificar as superfícies do presente trabalho como hidrofóbicas. Este resultado difere de outros autores que avaliaram a superfície de Ti G4 com objetivo de empregá-las em implantes dentários [1,4].

De acordo com DUBBIEL et al. [9] apenas classificar as superfícies como hidrofílica ou hidrofóbica não é o suficiente para determinar uma boa relação do material com o meio celular e permitir osseointegração do implante. $\mathrm{O}$ ângulo de contato mostra a molhabilidade de uma superfície sólida pela água na presença de uma fase gasosa. Infelizmente, essa situação não é encontrada em experimentos de cultura de células ou no interior do corpo humano.

Pode-se observar nas tabelas 1 e 2 que o aumento do tempo e da temperatura da solução ácida usada no tratamento da superfície das amostras aumentaram a energia da superfície, tanto do Ti G4 como do Ti Hard. Da mesma forma é possível observar que nos dois grupos houve também aumento nos parâmetros de rugosidade com o tratamento com ácido.

Analisando o gráfico da figura 2, observa-se que existe elevadíssima dependência da energia da superfície com as variáveis Ra, Rz, R3z, Rms, Pv, Pico, Vale e Srmax. Portanto, é extremamente importante a análise simultânea da influencia de todas as variáveis. Com o emprego das análises estatísticas usuais não foi possível quantificar a interdependência das variáveis e identificar a variável que exerce maior influência, deve-se empregar um modelo de "statistic learning". O emprego do modelo proposto permite selecionar a variável que apresenta maior influência. A interdependência dos parâmetros da rugosidade é uma das justificativas para que não se utilize somente um dos valores obtidos nas análises da rugosidade.

Uma das dificuldades nos estudos que comparam os tratamentos de superfície é a padronização das amostras. Um mesmo tratamento de superfície não produz duas superfícies idênticas em amostras com tamanhos de grão micrométricos e sub-micrométricos [10,11]. Pode-se observar nas tabelas 1 e 2 que o Ti Hard apresenta maior energia de superfície e possuem rugosidade diferente em relação ao Ti G4.

A análise de dados e com o emprego do algoritmo proposto no presente trabalho permite afirmar que, o fato do emprego do Ti Hard ou Ti G4, não existe influencia nos parâmetros de rugosidade ou molhabilidade. Isto é, se houvesse alguma forma de produzir superfícies com as mesmas morfologias das amostras com grãos micrométricos e sub-micrométrico, seria possível supor que as molhabilidades seriam semelhantes. Esta observação corrobora os resultados da literatura [11], segundo os quais a superfície do Ti Hard produzido por deformação plástica severa apresentaria diferentes fases e menores tamanhos de grãos que contribuem para o aumento na energia de superfície e na ósseointegração.

\section{CONCLUSÕES}

Com base nos resultados obtidos e na metodologia de análise de dados empregada, é possível concluir que:

a) A metodologia proposta de usar a técnica LASSO (Least Absolute Shrinkageand Selection Operator) associada à técnica de Double Selection permitiu obter uma correlação da molhabilidade da superfície com vários parâmetros da rugosidade.

b) existe uma correlação da influência entre os parâmetros da rugosidade na molhabilidade e os parâmetros Ra, PV e Rms são os que exercem maior influência no ângulo de contato e na energia da superfície;

c) o ângulo de contato aumenta com o aumento dos parâmetros que caracterizam a rugosidade de forma não linear;

d) o tamanho dos grãos do titânio (Ti G4 ou Ti Hard) não influenciou na molhabilidade das amostras.

\section{AGRADECIMENTOS}

Os autores agradecem ao CNPq, CAPES e FAPERJ pelo apoio financeiro. 


\section{BIBLIOGRAFIA}

[1] ELIAS, C.N. "Factors Affecting the Success of Dental Implants", In: Implant Dentistry - A Rapidly Evolving Practice, Ed by Ilser Turkyilmaz. Ed Rijeka In Tech, pp. 319-363 2011. DOI: 10.5772/18746

[2] MARTINEZ, E.F., ISHIKAWA, G.J., LEMOS, A.B., et al., "Evaluation of Ti surface treated with hydroxyapatite nanocrystals on osteoblastic cell behavior: an in vitro study", International Journal of Oral Maxillofacial Implants v.33, pp. 597-602, 2018.

[3] GONZÁlEZ-BLANCO, C., RIZO-GORRITA, M., LUNA-OLIVA, I., et al., "Human Osteoblast Cell Behaviour on Titanium Discs Treated with Argon Plasma", Materials, v, 12, pp. 1735, 2019.

[4] ELIAS, C.N., COUTINHO, M.P. "Rugosidade e molhabilidade do titânio usado em implantes dentários com superfícies tratadas", Revista Brasileira de Odontologia, v. 66, n. 2, pp. 234-8, jul./dez. 2009.

[5] RUSLAN V., SEMENOVA I.P., LATUYSH, V.V., et al., "Nanostructured Titanium for Biomedical Applications", Advanced Biomaterials, pp. B15-B17. DOI: 10.1002/adem.200800026.

[6] TIBSHIRANI, R. "Regression shrinkage and selection via the lasso", Journal of the Royal Statistical Society. Série B (Methodological) , pp. 267-288, 1996.

[7] BELLONI, A., CHERNOZHUKOV, V., HANSEN, C. "Inference on treatment effects after selection among high-dimensional controls", The Review of Economic Studies, v.81, n.2, pp. 608- 650, 2014.

[8] YAN, Y., CHIBOWSKI, E., SZCZÉS, A. "Surface properties of Ti-6Al-4V alloy part I: Surface roughness and apparent surface free energy", Materials Science and Engineering, v.70 pp. 207-215, 2017.

[9] DUBIEL, E.A., MARTIN, Y., VERMETTE, P. "Bridging the gap between physicochemistry and interpretation prevalent in cell-surface interactions", Chemistry Reviews, v.111, n.4, pp.2900-36, 2011.

[10] SERRA, G., MORAIS, L., ELIAS, C.N., et al., Nanostructured severe plastic deformation processed Ti for orthodontic mini-implants", Materials Science and Engineering C., v.33 pp.4197-4202, 2013.

[11] BAEK, S.M., SHIN, M.H., MOON, J., et al., "Superior Pre-Osteoblast Cell Response of Etched U1trafine-Grained Titanium with a Controlled Crystallographic Orientation", Scientific Reports v.7 pp. 44213$223,2017$.

\section{ORCID}

Gustavo Boehmer Leite Yuri Resende Fonseca

Alaelson Vieira Gomes Carlos Nelson Elias https://orcid.org/0000-0001-6111-0703

https://orcid.org/0000-0002-7364-5729

https://orcid.org/0000-0003-1651-2804 https://orcid.org/0000-0002-7560-6926 